\title{
FAMILIES OF EXOTIC AFFINE 3-SPHERES
}

\author{
ADRIEN DUBOULOZ
}

\begin{abstract}
We construct algebraic families of exotic affine 3-spheres, that is, smooth affine threefolds diffeomorphic to a non-degenerate smooth complex affine quadric of dimension 3 but non algebraically isomorphic to it. We show in particular that for every smooth topologically contractible affine surface $S$ with trivial automorphism group, there exists a canonical smooth family of pairwise non isomorphic exotic affine 3-spheres parametrized by the closed points of $S$.
\end{abstract}

\section{INTRODUCTION}

An exotic affine $n$-sphere is a smooth complex affine variety diffeomorphic to the standard affine algebraic $n$-sphere $\mathbb{S}^{n}=\left\{x_{1}^{2}+\cdots+x_{n+1}^{2}=1\right\}$ in $\mathbb{A}_{\mathbb{C}}^{n+1}$ but not algebraically isomorphic to it. In dimension $n=1$ or 2 , the algebraic structure on a smooth affine variety diffeomorphic to $\mathbb{S}^{n}$ is actually uniquely determined by its topology: $\mathbb{S}^{1} \simeq \mathbb{A}_{\mathbb{C}}^{1} \backslash\{0\}$ is the unique smooth affine curve $C$ with $H_{1}(C ; \mathbb{Z}) \simeq \mathbb{Z}$, and a smooth affine surface $S$ is algebraically isomorphic to $\mathbb{S}^{2}$ if and only if it has the same homology type and the same homotopy type at infinity as $\mathbb{S}^{2}$ (see [4. Theorem 3.3]). Examples of smooth affine threefolds with the same homology type as $\mathbb{S}^{3}$ were first constructed by D. Finston and S. Maubach in the form of total spaces of certain locally trivial $\mathbb{A}^{1}$-bundles over the smooth locus $S_{p, q, r}^{*}=S_{p, q, r} \backslash\{(0,0,0)\}$ of a Brieskorn surface

$$
S_{p, q, r}=\left\{x^{p}+y^{q}+z^{r}=0\right\} \subset \mathbb{A}^{3}, \quad 1 / p+1 / q+1 / r<1 .
$$

These threefolds are all diffeomorphic to each others, admitting the corresponding Brieskorn homology sphere $\Sigma(p, q, r)$ as a strong deformation retract. The main result of [5] asserts that their isomorphy types as abstract varieties are uniquely determined by their isomorphy classes as $\mathbb{A}^{1}$-bundles over $S_{p, q, r}^{*}$, up to composition by automorphisms of $S_{p, q, r}^{*}$. In particular, their construction gives rise to discrete families of exotic "homology" affine 3 -spheres (see Example 4).

Natural candidates of exotic affine 3 -spheres are the total spaces of nontrivial $\mathbb{A}^{1}$-bundles over the punctured affine plane $\mathbb{A}_{*}^{2}$. Indeed, noting that $\mathbb{S}^{3} \simeq \mathrm{SL}_{2}(\mathbb{C})=\{x v-y u=1\} \subset \mathbb{A}_{*}^{2} \times \mathbb{A}^{2}$, where $\mathbb{A}_{*}^{2}=$ $\operatorname{Spec}(\mathbb{C}[x, y]) \backslash\{(0,0)\}$, one checks more generally that all the varieties

$$
X_{m, n}=\left\{x^{m} v-y^{n} u=1\right\} \subset \mathbb{A}_{*}^{2} \times \mathbb{A}^{2}, \quad m+n \geq 2,
$$

are diffeomorphic to the trivial $\mathbb{R}^{2}$-bundle $\mathbb{R}^{2} \times \mathbb{A}_{*}^{2}$ over $\mathbb{A}_{*}^{2}$ when equipped with the euclidean topology. It was established in [4] that for $m+n>2, X_{m, n}$ is indeed an exotic affine 3 -sphere. But the classification of all threefolds $X_{m, n}, m+n>2$, up to isomorphism remains highly elusive, in particular, the techniques developed in loc. cit. do not allow to distinguish the $X_{m, n}, m+n>2$, from each others. As a consequence, all these threefolds could very well be all isomorphic, forming thus a unique class of exotic affine 3-spheres.

In this article, we build up on these ideas to construct infinitely many pairwise non isomorphic new exotic affine 3 -spheres, arising as total spaces of $\mathbb{A}^{1}$-bundles over 1-punctured smooth topologically contractible algebraic surfaces $S$. In particular, we obtain the following:

Theorem. For every smooth topologically contractible complex algebraic surface $S$, there exists a canonical smooth family $h: \mathcal{V} \rightarrow S$ of affine threefolds with the following properties:

a) The closed fibers are all diffeomorphic to $\mathbb{S}^{3}$,

b) The fibers of $h: \mathcal{V} \rightarrow S$ over two closed points $p$ and $p^{\prime}$ of $S$ are isomorphic if and only if $p$ and $p^{\prime}$ belong to the same orbit of the action of $\operatorname{Aut}(S)$ on $S$.

If $S=\mathbb{A}^{2}$, then the family $h: \mathcal{V} \rightarrow \mathbb{A}^{2}$ is actually isomorphic to the trivial one $\mathbb{S}^{3} \times \mathbb{A}^{2}$. But in contrast, if $S$ is a general smooth topologically contractible complex algebraic surface $S$ of non-negative Kodaira dimension $\kappa(S), \operatorname{Aut}(S)$ is trivial and the closed fibers of $h: \mathcal{V} \rightarrow S$ are pairwise non isomorphic exotic affine 3-spheres.

2000 Mathematics Subject Classification. 14R05, 14R25, 14J10.

This research was partialy funded by ANR Grant "BirPol" ANR-11-JS01-004-01. 


\section{Preliminaries}

Here we review a construction of Zariski locally trivial $\mathbb{A}^{1}$-bundles over the complement of certain closed subschemes of pure codimension 2 in a given scheme that we will use repeatedly in the article. We establish a criterion for isomorphism of total spaces of certain of these bundles.

1.1. Codimension 2 subschemes and locally trivial $\mathbb{A}^{1}$-bundles. Suppose given a scheme $Y$ and closed subscheme $Z \subset Y$ of pure codimension 2 with ideal sheaf $\mathcal{I}_{Z} \subset \mathcal{O}_{Y}$ for which we have a locally free resolution

$$
0 \longrightarrow \mathcal{L} \stackrel{a}{\longrightarrow} \mathcal{F} \stackrel{b}{\longrightarrow} \mathcal{I}_{Z} \longrightarrow 0,
$$

where $\mathcal{L}$ and $\mathcal{F}$ are locally free sheaves on $Y$, of rank 1 and 2 respectively. In particular, $Z$ is a local complete intersection in $Y$. We let $\bar{q}: X=\mathbb{P}(\mathcal{F}) \rightarrow Y$ be the relative Proj of the symmetric algebra $\operatorname{Sym}_{Y} \mathcal{F}$ of $\mathcal{F}$ and we let $H \subset X$ be the closed subscheme determined by the surjection $b: \mathcal{F} \rightarrow \mathcal{I}_{Z}$. We let $V_{Z}=X \backslash H$ and we let $p: L=\operatorname{Spec}_{Y \backslash Z}\left(\left.\operatorname{Sym}^{\cdot} \mathcal{L}^{-1}\right|_{Y \backslash Z}\right) \rightarrow Y \backslash Z$ be the restriction to $Y \backslash Z$ of the total space of the invertible sheaf $\mathcal{L}$.

Proposition 1. The scheme $V_{Z}$ is affine over $Y$ and $\left.\bar{q}\right|_{V_{Z}}$ factors through an L-torsor $\rho_{Z}: V_{Z} \rightarrow Y \backslash Z$ whose isomorphy class in $H^{1}(Y \backslash Z, L) \simeq \operatorname{Ext}_{Y \backslash Z}^{1}\left(\mathcal{O}_{Y \backslash Z}, \mathcal{L}\right)$ coincide with that of the restriction

$$
\left.\left.\left.0 \longrightarrow \mathcal{L}\right|_{Y \backslash Z} \stackrel{a}{\longrightarrow} \mathcal{F}\right|_{Y \backslash Z} \stackrel{b}{\longrightarrow} \mathcal{I}_{Z}\right|_{Y \backslash Z} \simeq \mathcal{O}_{Y \backslash Z} \longrightarrow 0
$$

of the exact sequence (1.1) to $Y \backslash Z$. .

Proof. By definition, $H$ is equal to the closed subscheme $\operatorname{Proj}_{Y}\left(\operatorname{Sym}^{\prime} \mathcal{I}_{Z}\right)$ of $X$, and since $Z$ is a local complete intersection of codimension 2 in $Y$, $\operatorname{Sym}_{Y} \mathcal{I}_{Z}$ is locally isomorphic as a sheaf of graded $\mathcal{O}_{Y}$-algebra to the Rees $\mathcal{O}_{Y \text {-algebra }} \bigoplus_{n>0} \mathcal{I}_{Z}^{n}$ of $\mathcal{I}_{Z}$. So locally over $Y,\left.\bar{q}\right|_{H}: H \rightarrow Y$ is isomorphic to the blow-up of $Y$ along $Z$, implying in particular that $\bar{q}^{-1}(Z)$ is fully contained in $H$ while $\left.H\right|_{X \backslash \bar{q}^{-1}(Z)}$ is a section of the restriction of the locally trivial $\mathbb{P}^{1}$-bundle $\bar{q}: X \rightarrow Y$ over $Y \backslash Z$. As a consequence, the image of $q$ is equal to $Y \backslash Z$ and the corestriction $\rho_{Z}: V_{Z} \rightarrow Y \backslash Z$ of $\left.\bar{q}\right|_{V_{Z}}$ to its image is a locally trivial $\mathbb{A}^{1}$-bundle. Since $H$ is relatively ample over $Y, V_{Z}=X \backslash H$ is affine over $Y$, and the fact that $\rho_{Z}: V_{Z} \rightarrow Y \backslash Z$ is actually an $L$-torsor with the given isomorphy class follows directly from the construction.

Example 2. In the case where $Z$ is a global complete intersection in $Y$, the above construction applied to a free resolution

$$
0 \longrightarrow \mathcal{O}_{Y} \stackrel{{ }^{t}(-g, f)}{\longrightarrow} \mathcal{O}_{Y}^{\oplus 2} \longrightarrow{ }^{(f, g)} \longrightarrow \mathcal{I}_{Z} \longrightarrow 0
$$

for some global regular functions $f, g \in \Gamma\left(Y, \mathcal{O}_{Y}\right)$ such that $Z=V(f, g)$ yields a scheme $X$ is isomorphic to $Y \times \mathbb{P}_{[u: v]}^{1}$, for which $H$ is equal to the closed subscheme $\{f v-g u=0\}$. The morphism

$$
V_{Z}=X \backslash H \rightarrow Y \times \operatorname{Spec}(\mathbb{C}[U, V]), \quad(y,[u: v]) \mapsto\left(y, \frac{u}{f v-g u}, \frac{v}{f v-g u}\right)
$$

then induces an isomorphism between $V_{Z}$ and the closed sub-subscheme of $Y \times \operatorname{Spec}(\mathbb{C}[\mathrm{U}, \mathrm{V}])$ with equation $f V-g U=1$. Note that if

$$
0 \longrightarrow \mathcal{O}_{Y} \stackrel{{ }^{t}\left(-g^{\prime}, f^{\prime}\right)}{\longrightarrow} \mathcal{O}_{Y}^{\oplus 2} \stackrel{\left(f^{\prime}, g^{\prime}\right)}{\longrightarrow} \mathcal{I}_{Z} \longrightarrow 0
$$

is another free resolution, then there exists $\varphi=\left(\begin{array}{ll}a & b \\ c & d\end{array}\right) \in \mathrm{GL}_{2}\left(\Gamma\left(Y, \mathcal{O}_{Y}\right)\right)$ such that $f^{\prime}=a f+b g$ and $g^{\prime}=c f+d g$. This element $\varphi$ determines in turn an $Y$-automorphism $(U, V) \mapsto(-c U+a V, d U-b V)$ of $Y \times \operatorname{Spec}(\mathbb{C}[U, V])$ which maps $\left\{f^{\prime} V-g^{\prime} U=1\right\}$ isomorphically onto $\{f V-g U=1\}$.

1.2. A criterion for isomorphism. The following proposition provides a criterion to decide when the total spaces of certain torsors $\rho_{Z}: V_{Z} \rightarrow Y \backslash Z$ and $\rho_{Z^{\prime}}: V_{Z^{\prime}} \rightarrow Y^{\prime} \backslash Z^{\prime}$ as above are isomorphic as abstract algebraic varieties.

Proposition 3. Let $(Y, Z)$ and $\left(Y^{\prime}, Z^{\prime}\right)$ be pairs consisting of a normal affine variety and a scheme theoretic global complete intersection of pure codimension 2 , and let $\rho_{Z}: V_{Z} \rightarrow Y \backslash Z$ and $\rho_{Z^{\prime}}: V_{Z^{\prime}} \rightarrow Y^{\prime} \backslash Z^{\prime}$ be the corresponding varieties. If either $Y \backslash Z$ or $Y^{\prime} \backslash Z$ has non-negative Kodaira dimension, then $V_{Z}$ and $V_{Z^{\prime}}$ are isomorphic as abstract varieties if and only if the pairs $(Y, Z)$ and $\left(Y^{\prime}, Z^{\prime}\right)$ are isomorphic. 
Proof. Given an isomorphism $\varphi: Y \rightarrow Y^{\prime}$ mapping $Z$ onto $Z^{\prime}$, it follows from the construction of $V_{Z}$ and $V_{Z^{\prime}}$ that $V_{Z}$ and $V_{Z^{\prime}} \times_{S^{\prime}} S \simeq V_{Z^{\prime}}$ are isomorphic as schemes over $Y$. Conversely, if either $\kappa(Y \backslash Z)$ or $\kappa\left(Y^{\prime} \backslash Z^{\prime}\right)$ is non-negative, then it follows from a straightforward adaptation of the proof of the Iitaka-Fujita Strong Cancellation Theorem [11] to the case of locally trivial $\mathbb{A}^{1}$-bundles, that every isomorphism $\Psi: V_{Z} \rightarrow V_{Z^{\prime}}$ descends to a unique isomorphism $\psi: Y \backslash Z \rightarrow Y^{\prime} \backslash Z^{\prime}$ for which the following diagram is commutative

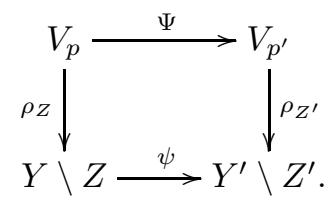

Since $Y$ and $Y^{\prime}$ are affine and normal, and $Z$ and $Z^{\prime}$ have pure codimension 2 in $Y$ and $Y^{\prime}$ respectively, $\psi$ uniquely extends to an isomorphism $\varphi: Y \rightarrow Y^{\prime}$ such that $\varphi\left(Z_{\text {red }}\right)=Z_{\text {red }}^{\prime}$. Let $Z=V(f, g), Z^{\prime}=V\left(f^{\prime}, g^{\prime}\right)$ and let $Z^{\prime \prime}=\varphi^{-1}\left(Z^{\prime}\right)=V\left(f^{\prime \prime}, g^{\prime \prime}\right)$ where $f^{\prime \prime}=f^{\prime} \circ \varphi$ and $g^{\prime \prime}=g^{\prime} \circ \varphi$. The isomorphism $\Psi$ factors through an isomorphism $\tilde{\Psi}: V_{Z} \rightarrow V_{Z^{\prime \prime}} \simeq V_{Z^{\prime}} \times_{Y^{\prime}} Y$ of locally trivial $\mathbb{A}^{1}$-bundles over $Y \backslash Z$. Now $V_{Z}$ and $V_{Z^{\prime \prime}}$ are isomorphic as locally trivial $\mathbb{A}^{1}$-bundles over $Y_{*}=Y \backslash Z=Y \backslash Z^{\prime \prime}$ if and only if there exists an isomorphism of extensions

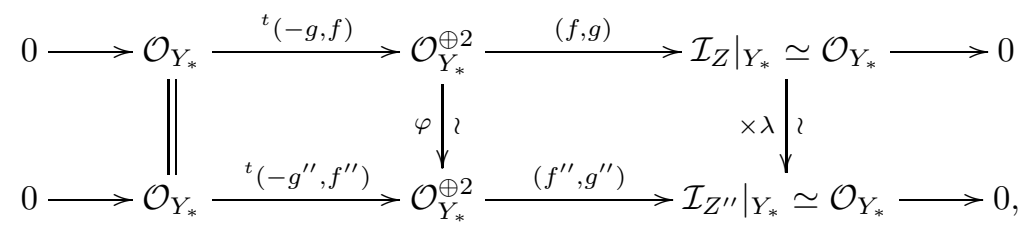

where the isomorphism on the middle is given by an element $\varphi=\left(\begin{array}{cc}a & b \\ c & d\end{array}\right) \in \mathrm{GL}_{2}\left(\Gamma\left(Y_{*}, \mathcal{O}_{Y_{*}}\right)\right)=\mathrm{GL}_{2}\left(\Gamma\left(Y, \mathcal{O}_{Y}\right)\right)$ and the isomorphism on the right-hand side is the multiplication by an element $\lambda \in \Gamma\left(Y_{*}, \mathcal{O}_{Y_{*}^{*}}^{*}\right)=\Gamma\left(Y, \mathcal{O}_{Y}^{*}\right)$. So $\mathcal{I}_{Z^{\prime \prime}}=((a f+b g),(c f+d g))=(\lambda f, \lambda g)=\mathcal{I}_{Z}$, which implies that $\varphi(Z)=Z^{\prime}$ as desired.

Example 4. (See [5, Example 1]) Since the smooth locus $S_{p, q, r}^{*}=S_{p, q, r} \backslash\{(0,0,0)\}$ of a Brieskorn surface $S_{p, q, r}=\left\{x^{p}+y^{q}+z^{r}=0\right\}$ in $\mathbb{A}^{3}$, where $p, q, r$ are pairwise relatively prime and $1 / p+1 / q+1 / r<1$, has Kodaira dimension equal to $1\left[10\right.$, it follows from Proposition 3 that the total spaces of the $\mathbb{A}^{1}$-bundles

$$
\rho_{m, n}: X_{m, n}=\left\{x^{m} V-y^{n} U=1\right\} \rightarrow S_{p, q, r}^{*}, \quad m, n \geq 1
$$

associated to the closed subschemes $Z_{m, n} \subset S_{p, q, r}$ with defining ideals $I_{m, n}=\left(x^{m}, y^{n}\right), m, n \geq 1$ are isomorphic if and only if the corresponding pairs $\left(S_{p, q, r}, Z_{m, n}\right)$ are isomorphic. Since $\operatorname{Aut}\left(S_{p, q, r}\right)$ is isomorphic to the multiplicative group $\mathbb{G}_{m}$, acting linearly on $S_{p, q, r}$ by $\lambda \cdot(x, y, z)=\left(\lambda^{q r} x, \lambda^{p r} y, \lambda^{q r} z\right)$, two such pairs $\left(S_{p, q, r}, Z_{m, n}\right)$ and $\left(S_{p, q, r}, Z_{m^{\prime}, n^{\prime}}\right)$ are isomorphic if and only if $(m, n)=\left(m^{\prime}, n^{\prime}\right)$, and so the varieties $X_{m, n}$ are pairwise non isomorphic.

Remark 5. In Proposition 3, the hypothesis on the Kodaira dimension is crucial. For instance, let $\mathbb{A}^{2}=$ $\operatorname{Spec}(\mathbb{C}[x, y])$ and consider the $\mathbb{A}^{1}$-bundles

$$
\rho_{m, n}: X_{m, n}=\left\{x^{m} V-y^{n} U=1\right\} \rightarrow \mathbb{A}^{2} \backslash\{(0,0)\}
$$

associated to the closed subschemes $Z_{m, n} \subset \mathbb{A}^{2}$ with defining ideals $I_{m, n}=\left(x^{m}, y^{n}\right), m, n \geq 1$. It is not difficult to check that two pairs $\left(\mathbb{A}^{2}, Z_{m, n}\right)$ and $\left(\mathbb{A}^{2}, Z_{m^{\prime}, n^{\prime}}\right)$ are isomorphic if and only if $\{m, n\}=\left\{m^{\prime}, n^{\prime}\right\}$ (see e.g. [4, Proposition 2.2]). On the other hand, it was established in [4, Theorem 2.3] that if $m+n=m^{\prime}+n^{\prime}$ then $X_{m, n}$ and $X_{m^{\prime}, n^{\prime}}$ are isomorphic as abstract varieties.

\section{ALGEBRAIC FAMILIES OF EXOTIC 3-SPHERES OVER PUNCTURED CONTRACTIBLE SURFACES}

In this section, we construct algebraic families of affine 3 -spheres in the form of locally trivial $\mathbb{A}^{1}$-bundles over 1-punctured topologically contractible affine surfaces. Recall that a topological manifold is called contractible if it has the homotopy type of a point. A smooth complex algebraic variety is called contractible if it is so when equipped with its underlying structure of real topological manifold. 
2.1. Basic properties of contractible surfaces. Smooth contractible algebraic surfaces have been intensively studied during the last decades. We just recall a few basic facts about these, referring the reader to [20, § 2] and [13, Chapter 3] and the references therein for a complete overview. First of all, these surfaces are all affine and rational. They are partially classified in terms of their (logarithmic) Kodaira dimension $\kappa: \mathbb{A}^{2}$ is the only such surface of negative Kodaira dimension, there is no contractible surface of Kodaira dimension 0, contractible surfaces of Kodaira dimension 1 are fully classified in terms of the structure of their smooth completions. So far, there is no classification of contractible surfaces of Kodaira dimension 2, but many families of examples have been constructed [2, 7, 15, 18.

The Picard group of a smooth contractible surface $S$ is trivial [13, Lemma 4.2.1], and more generally, the affineness and the rationality of $S$ combined with a result of Murthy [16] imply that every locally free sheaf $\mathcal{E}$ of rank $r \geq 2$ on $S$ is free. Of particular interest for us is the following consequence of these facts:

Lemma 6. In a smooth contractible affine surface $S$, every closed subscheme $Z \subset S$ of pure dimension which is a scheme theoretic local complete intersection is a scheme theoretic global complete intersection.

In particular, every closed point $p$ on a smooth contractible surface $S$ is a scheme theoretic global complete intersection.

Proof. Since the Picard group $\operatorname{Pic}(S)$ is trivial, every scheme theoretic local complete intersection of pure codimension 1 is a global complete intersection. If $Z$ has pure codimension 2 , hence pure dimension 0 , then its normal sheaf $\mathcal{N}_{Z / S}$ is locally free of rank 2 , hence isomorphic to the trivial sheaf $\mathcal{O}_{Z}^{\oplus 2}$. So $\Lambda^{2} \mathcal{N}_{Z / S} \simeq \mathcal{O}_{Z}$, and it follows from the Serre construction [19] (see also [1, Theorem 1.1]) that there exists an exact sequence $0 \rightarrow \mathcal{O}_{S} \rightarrow \mathcal{E} \rightarrow \mathcal{I}_{Z} \rightarrow 0$, where $\mathcal{E}$ is locally free of rank 2, hence free of rank 2, providing an isomorphism $Z \simeq V(f, g)$ for some regular function $f, g \in \Gamma\left(S, \mathcal{O}_{S}\right)$.

We also record the following result concerning automorphism groups of smooth contractible surfaces:

Proposition 7. The automorphism group of a contractible surface $S$ different from $\mathbb{A}^{2}$ is either trivial if $\kappa(S)=1$ or finite if $\kappa(S)=2$.

Proof. The case where $\kappa(S)=2$ follows from the more general fact that any variety of maximal Kodaira dimension has finite automorphism group (see e.g. [9, Theorem 6]). For the case $\kappa(S)=1$, see [17].

Remark 8. It is established in [17] that certain smooth contractible surfaces $S$ with $\kappa(S)=2$ such as the Ramanujam surface [18] and the Gurjar-Miyanishi surfaces [7] have a trivial automorphism group. But there exists examples of smooth contractible surfaces of log-general type with nontrivial finite automorphism groups (see e.g. [15, 2]).

2.2. A construction of affine 3-spheres. Let $S$ be a smooth contractible surface and let $Z=V(f, g) \subset S$ be a global scheme theoretic complete intersection whose support is a closed point $p$ of $S$. By applying the construction described in $\$ 1.1$ to the corresponding resolution

$$
0 \longrightarrow \mathcal{O}_{S} \stackrel{{ }^{t}(-g, f)}{\longrightarrow} \mathcal{O}_{S}^{\oplus 2} \longrightarrow{ }^{(f, g)} \longrightarrow \mathcal{I}_{Z} \longrightarrow 0
$$

of the ideal $\mathcal{I}_{Z} \subset \mathcal{O}_{S}$ of $Z$, we obtain a smooth affine threefold

$$
V_{Z} \simeq\{f V-g U=1\} \subset S \times \mathbb{A}^{2}
$$

equipped with the structure of a locally trivial $\mathbb{G}_{a}$-bundle $\rho_{Z}=\operatorname{pr}_{S}: V_{Z} \rightarrow S \backslash Z=S \backslash\{p\}$ for the fixed point free $\mathbb{G}_{a, S \backslash\{p\}}$-action defined by $t \cdot(s, U, V)=(s, U+f(s) t, V+g(s) t)$.

Example 9. Since by Lemma 6 every closed point $p$ on a smooth contractible surface $S$ is a global scheme theoretic complete intersection $p=V(f, g)$ for some $f, g \in \Gamma\left(S, \mathcal{O}_{S}\right)$, we obtain in particular for every such point $p$ a smooth affine threefold

$$
\rho_{p}: V_{p}(S) \simeq\{f V-g U=1\} \rightarrow S \backslash\{p\},
$$

whose isomorphy class as a scheme over $S \backslash\{p\}$ is independent on the choice of the two generators of the ideal $I_{p}$ of $p$ in $\Gamma\left(S, \mathcal{O}_{S}\right)$ (see Example 2).

The variety $V_{o}\left(\mathbb{A}^{2}\right)$ corresponding to the origin $o=(0,0)$ in $\mathbb{A}^{2}=\operatorname{Spec}(\mathbb{C}[x, y])$ is isomorphic to $\mathrm{SL}_{2}(\mathbb{C})=$ $\{x V-y U=1\}$, hence to the standard affine 3 -sphere $\mathbb{S}^{3}$. In particular, it is diffeomorphic to $\mathbb{S}^{3}$. More generally, we have the following result: 
Proposition 10. For every pair $(S, Z)$ consisting of a smooth contractible surface $S$ and a global scheme theoretic complete intersection $Z \subset S$ supported at a closed point $p$ of $S$, the smooth affine threefold $V_{Z}$ is diffeomorphic to the standard affine 3 -sphere $\mathbb{S}^{3}=\left\{x_{1}^{2}+x_{2}^{2}+x_{3}^{2}+x_{4}^{2}=1\right\}$ in $\mathbb{A}^{4}$.

Proof. Since the sheaf $\mathcal{C}^{\infty}(S \backslash\{p\}, \mathbb{C})$ of complex valued $\mathcal{C}^{\infty}$-functions on $S \backslash\{p\}$ is soft (see e.g. [6. Theorem 5, p.25] ), every algebraic Cech 1-cocycle representing the class of the $\mathbb{G}_{a, S \backslash\{p\}}$-bundle $\rho_{Z}: V_{Z} \rightarrow S \backslash Z=S \backslash\{p\}$ in $H^{1}\left(S \backslash\{p\}, \mathcal{O}_{S \backslash\{p\}}\right)$ on some Zariski open covering of $S \backslash\{p\}$ is a coboundary when considered as a 1-cocycle with values in $\mathcal{C}^{\infty}(S \backslash\{p\}, \mathbb{C})$. So $\rho_{Z}: V_{Z} \rightarrow S \backslash\{p\}$ is diffeomorphic to the trivial $\mathbb{R}^{2}$-bundle over $S \backslash\{p\}$, and hence $V_{Z}$ is diffeomorphic to the complement in the trivial $\mathbb{R}^{2}$-bundle $\operatorname{pr}_{1}: S \times \mathbb{R}^{2} \rightarrow S$ of $\operatorname{pr}_{1}^{-1}(p) \simeq \mathbb{R}^{2}$. Since $S$ is contractible, $S \times \mathbb{R}^{2}$ is diffeomorphic to $\mathbb{R}^{6}$ by virtue of [12]. The assertion then follows from the fact that any two smooth closed embeddings of $\mathbb{R}^{2}$ into $\mathbb{R}^{6}$ are properly homotopic, and that in this range of dimensions any two properly homotopic smooth closed embeddings are ambiently isotopic [8, Chapter 8].

In particular, we obtain the following:

Corollary 11. For every pair $(S, Z)$ consisting of a smooth contractible surface $S$ of non-negative Kodaira dimension and a global scheme theoretic complete intersection $Z \subset S$ supported at a point, the corresponding threefold $V_{Z}$ is an exotic affine 3-sphere.

Proof. This follows from Proposition 3 applied to the pairs $(Y, Z)=(S, Z)$ and $\left(Y^{\prime}, Z^{\prime}\right)=\left(\mathbb{A}^{2},\{(0,0\})\right.$.

Corollary 12. Let $S$ and $S^{\prime}$ be non-isomorphic smooth contractible surface of non-negative Kodaira dimension. Then for every pair of closed points $p \in S$ and $p^{\prime} \in S^{\prime}$, the threefolds $V_{p}(S)$ and $V_{p^{\prime}}\left(S^{\prime}\right)$ are non-isomorphic exotic affine 3-spheres.

Proof. This follows again from Proposition 3 applied to the pairs $(S, p)$ and $\left(S^{\prime}, p^{\prime}\right)$.

Applying Proposition 3 to pairs of closed points on a fixed smooth contractible surface, we obtain:

Corollary 13. Let $S$ be a smooth contractible surface of non-negative Kodaira dimension and let $p, p^{\prime}$ be closed point on $S$. Then $V_{p}(S)$ and $V_{p^{\prime}}(S)$ are isomorphic as abstract algebraic varieties if and only if $p$ and $p^{\prime}$ belong to the same orbit of the action of $\operatorname{Aut}(S)$ on $S$.

2.3. Algebraic families of exotic affine 3-spheres. Given a smooth contractible surface $S$, we let $\Delta \subset$ $S \times S$ be the diagonal, we let $T=S \times S \backslash \Delta$ and we denote by $\pi: T \rightarrow S$ the restriction of the first projection. Since the normal sheaf $\mathcal{N}_{\Delta / S \times S} \simeq \mathcal{T}_{S}$ of $\Delta \simeq S$ in $S \times S$ is trivial, it follows from the Serre construction [1, Theorem 1.1] that the ideal sheaf $\mathcal{I}_{\Delta}$ admits a locally free resolution of the form

$$
0 \longrightarrow \mathcal{O}_{S \times S} \longrightarrow \mathcal{F} \longrightarrow \mathcal{I}_{\Delta} \longrightarrow 0
$$

for some locally free sheaf $\mathcal{F}$ of rank 2 on $S \times S$, uniquely determined up to isomorphism. Furthermore, the above extension can be chosen in such a way that its class in $\operatorname{Ext}_{S \times S}^{1}\left(\mathcal{I}_{\Delta}, \mathcal{O}_{S \times S}\right)$ coincides via the isomorphism $\operatorname{Ext}_{S \times S}^{1}\left(\mathcal{I}_{\Delta}, \mathcal{O}_{S \times S}\right) \simeq H^{0}\left(S \times S, \operatorname{det} \mathcal{N}_{\Delta / S \times S}\right)$ to the constant global section 1 of $\operatorname{det} \mathcal{N}_{\Delta / S \times S} \simeq \mathcal{O}_{S}$. We let $\rho_{\Delta}: \mathcal{V}_{\Delta} \rightarrow T$ be the $\mathbb{G}_{a}$-bundle over $T$ corresponding to this locally free resolution by the construction of 1.1 and we let $h=\pi \circ \rho_{\Delta}: \mathcal{V}_{\Delta} \rightarrow S$

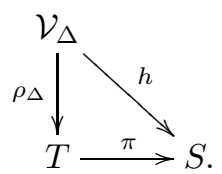

Proposition 14. With the notation above, the following hold:

a) $\mathcal{V}_{\Delta}$ is a smooth affine variety of dimension 5 .

b) The morphism $h=\pi \circ \rho_{\Delta}: \mathcal{V}_{\Delta} \rightarrow S$ is smooth and surjective.

c) For every closed point $j:\{p\} \hookrightarrow S$, we have commutative diagram with cartesian squares

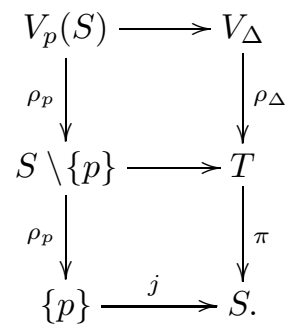


Proof. Since $\mathcal{V}_{\Delta}$ is affine over $S \times S$ by Proposition 1 and $S \times S$ is affine, $\mathcal{V}_{\Delta}$ is an affine variety. The smoothness and the surjectivity of $h$ follow from those of $\rho_{\Delta}: \mathcal{V}_{\Delta} \rightarrow T$ and $\pi: T \rightarrow S$. By construction, the scheme theoretic fiber $T_{p}$ of $\pi$ over a closed point $p \in S$ is isomorphic to $S \backslash\{p\}$ while the restriction of the exact sequence 2.1 to the fiber $(S \times S)_{p} \simeq S$ of $\operatorname{pr}_{1}: S \times S \rightarrow S$ over $p$ is a free resolution

$$
0 \longrightarrow \mathcal{O}_{S} \longrightarrow \mathcal{O}_{S}^{\oplus 2} \longrightarrow \mathcal{I}_{p} \longrightarrow 0
$$

of the ideal sheaf $\mathcal{I}_{p} \subset \mathcal{O}_{S}$ of $p$. This implies in turn that $\mathcal{V}_{\Delta, p} \simeq V_{p}(S)$.

Remark 15. When $S$ is different from $\mathbb{A}^{2}$, it seems to be unknown whether the middle locally free sheaf $\mathcal{F}$ occurring in the exact sequence 2.1 is free or not. If not free, then $\mathcal{F}$ would provide a counter-example to the Generalized Serre Problem on projective modules on topologically contractible affine varieties, and $S \times S$ would not be contractible in the unstable $\mathbb{A}^{1}$-homotopy category of Morel-Voevodsky.

For $S=\mathbb{A}^{2}=\operatorname{Spec}\left(\mathbb{C}\left[x_{1}, y_{1}\right]\right)$, the ideal $I_{\Delta}$ of the diagonal in $\mathbb{A}^{2} \times \mathbb{A}^{2}=\operatorname{Spec}\left(\mathbb{C}\left[x_{1}, y_{1}\right]\left[x_{2}, y_{2}\right]\right)$ is generated by the polynomials $x_{2}-x_{1}$ and $y_{2}-y_{1}$, and the corresponding variety $\rho_{\Delta}: \mathcal{V}_{\Delta} \rightarrow \mathbb{A}^{2} \times \mathbb{A}^{2} \backslash \Delta$ is thus isomorphic to the subvariety $\mathcal{W}$ of $\mathbb{A}^{2} \times \mathbb{A}^{2} \times \operatorname{Spec}(\mathbb{C}[U, V])$ defined by the equation $\left(x_{2}-x_{1}\right) V-\left(y_{2}-y_{1}\right) U=1$, equipped with the restriction of the projection onto $\mathbb{A}^{2} \times \mathbb{A}^{2}$. It follows in turn that the family $h: \mathcal{V}_{\Delta} \rightarrow \mathbb{A}^{2}$ is isomorphic to the trivial one $\mathbb{A}^{2} \times \mathrm{SL}_{2}(\mathbb{C})$ via the morphism

$$
\Phi: \mathcal{W} \rightarrow \mathbb{A}^{2} \times \mathrm{SL}_{2}(\mathbb{C}),\left(x_{1}, y_{1}, x_{2}, y_{2}, U, V\right) \mapsto\left(\left(x_{1}, y_{1}\right),\left(x_{2}-x_{1}\right),\left(y_{2}-y_{1}\right), U, V\right) .
$$

In contrast, when $S$ has non-negative Kodaira dimension, it follows from Corollary 13 that the fibers $V_{p}(S)$ and $V_{p^{\prime}}(S)$ of $h: \mathcal{V}_{\Delta} \rightarrow S$ over two closed point $p$ and $p^{\prime}$ of $S$ are isomorphic if and only if $p$ and $p^{\prime}$ belong to the same orbit of the action of $\operatorname{Aut}(S)$ on $S$. Since there does not exist any smooth contractible surface of Kodaira dimension 0, we have $\kappa(S) \geq 1$ necessarily, and since by Proposition 7 the action of Aut $(S)$ on $S$ is not transitive, $h: \mathcal{V}_{\Delta} \rightarrow S$ is not isomorphic to the trivial family $S \times \mathrm{SL}_{2}(\mathbb{C})$. In fact, as a consequence of Proposition 14 c) and Corollary 11, we have the following stronger result:

Theorem 16. Let $S$ be a smooth contractible surface of non-negative Kodaira dimension with trivial automorphism group. Then $h: \mathcal{V}_{\Delta} \rightarrow S$ is a smooth family of pairwise non isomorphic exotic affine 3 -spheres.

\section{REFERENCES}

1. E. Arrondo, A home-made Hartshorne-Serre correspondence, Rev. Mat. Complut. 20 (2007), no. 2, 423-443.

2. T. tom Dieck, Symmetric homology planes, Math. Ann. 286 (1990), no. 1-3, 143-152.

3. T. tom Dieck and T. Petrie, Contractible affine surfaces of Kodaira dimension one, Japan J. Math. 16 (1990), 147-169.

4. A. Dubouloz, D.R. Finston, On exotic affine 3-spheres, J. Algebraic Geom. 23 (2014), no. 3, 445-469.

5. D.R. Finston and S. Maubach, The automorphism group of certain factorial threefolds and a cancellation problem, Israel J. Math. 163 (2008), 369-381.

6. H. Grauert and R. Remmert, Theory of Stein spaces, Springer Verlarg, 1979.

7. R.V. Gurjar and M. Miyanishi, Affine surfaces with $\bar{\kappa} \leq 1$, Algebraic Geometry and Commutative Algebra, in honor of M. Nagata, 1987, 99-124.

8. M. W. Hirsch, Differential topology, Springer-Verlag, New York, 1976, Graduate Texts in Mathematics, No. 33.

9. S. Iitaka, On logarithmic Kodaira dimensions of algebraic varieties, Complex Analysis and Algebraic geometry, Iwanami, Tokyo, 1977, 175-189.

10. S. Iitaka, Homogeneous Lüroth theorem and logarithmic classification of algebraic surfaces, Proc. Kinosaki Symp. on algebraic geometry, 1977.

11. S. Iitaka, T. Fujita, Cancellation theorem for algebraic varieties, J. Fac. Sci. Univ. Tokyo, Sect.IA, 24 (1977), 123-127.

12. D. R. McMillan and E. C. Zeeman, On contractible open manifolds, Proc. Cambridge Philos. Soc. 58 (1962), $221-224$.

13. M. Miyanishi, Open Algebraic Surfaces, CRM Monogr. Ser., 12, Amer. Math. Soc., Providence, RI, 2001.

14. M. Miyanishi and T. Sugie, Examples of homology planes of general type, J. Math. Kyoto Univ. 30 (1990), 337-342.

15. M. Miyanishi and T. Sugie, $\mathbb{Q}$-homology planes with $\mathbb{C}^{* *}$-fibrations, Osaka J. Math. 28 (1991), 1-26.

16. M. P. Murthy, Vector Bundles Over Affine Surfaces Birationally Equivalent to a Ruled Surface, Annals of Maths. vol. 89, No. 2 (1969), 242-253.

17. T. Petrie, Algebraic automorphisms of smooth affine surfaces, Invent. Math. 95 (1989), 355-378.

18. C.P. Ramanujam, A topological characterization of the affine plane as an algebraic variety, Ann. of Maths. 94 (1971), 69-88.

19. J.P. Serre, Sur les modules projectifs, Séminaire Dubreil-Pisot (1960/61), Secr. Math. Paris, exposé 2, (1961).

20. M. Zaidenberg, Exotic algebraic structures on affine spaces (Russian) Algebra i Analiz 11 (1999), no. 5, 3-73; translation in St. Petersburg Math. J. 11 (2000), no. 5, 703-760.

imB UMr5584, CNRS, Univ. Bourgogne Franche-Comté, F-21000 Dijon, France.

E-mail address: adrien.dubouloz@u-bourgogne.fr 